\title{
Mobility-Aware Node Clustering with Fuzzy Logic for Wireless Mesh Network
}

\author{
Tapodhir Acharjee ${ }^{1}$ and Sudipta Roy ${ }^{2}$ \\ ${ }^{1,2}$ Department of Computer Science \& Engineering \\ Assam University, Silchar-788011 \\ Assam, India \\ 1tapacharjee@gmail.com, ${ }^{2}$ sudipta.it@gmail.com
}

\begin{abstract}
In recent times, Wireless Mesh Network (WMN) has received prominence worldwide due to the evolution of wireless networks as a ubiquitous and seamless broadband service provider. Routing on WMNs is one of the most prominent research issues on these days. Hierarchical clustering based routing protocols are proposed by the researchers for large networks. In these protocols, all the available nodes are grouped into clusters. Each cluster consists of cluster members, cluster head and gateway nodes where cluster head plays the most vital role to form an efficient cluster. Selection of a better cluster head depends on the decisions made from different parameters and their relations. In this situation, fuzzy logic suits better by providing better analysis and helps to make the right decision based on different parameter values and their relations. In this paper, a fuzzy logic based hierarchical clustering technique is proposed for WMN. The proposed technique along with the existing Fuzzy Logic Control Clustering Algorithm(FLCCA) are experimented using the simulation software NS3 and the simulated results are compared to establish the efficiency of the proposed technique.
\end{abstract}

Keywords: Wireless Mesh Network; Mobile Ad-hoc Network; Cluster Head; Cluster Based Routing;Fuzzy Logic

\section{Introduction}

Wireless Mesh Network (WMN) is established as one of the most significant wireless technology for several applications, like broadband home networking, community and neighborhood networking, battlefield surveillance, Voice over IP(VoIP), intelligent transportation systems etc. Most importantly, it is gaining more attention as a tool for Internet service providers (ISPs) and the end-users to access efficient wireless broadband service at minimum cost. WMNs have the ability to self-organize and self-conFigure dynamically because the nodes in the network are capable of establishing automatically an ad hoc network with mesh connectivity [1]. There are three different kinds of mesh architecture, namely, Infrastructure WMNs, Client WMNs and Hybrid WMNs. This classification is based on the fact: whether the mesh clients are having any infrastructure created by mesh routers or not or it is the combination of both(hybrid) [2].

Scalable routing protocols are needed for providing last mile connectivity in WMNs. For scalable networks, Hierarchical routing technique produces good results as only a few of the nodes are involved in routing [3-4]. With the help of efficient clustering techniques, the important routing performance parameters like, throughput, bandwidth consumption, energy consumption, packet delivery ratio, end to end delay may be improved [5]. Efficient clustering protocols try to solve different design goals for the application they are designed for.

Clusterhead selection is a complex decision making process involving various parameters which are qualitative, inexact, or uncertain in nature. It is found that fuzzy logic can 
produce better results when uncertainty is considered. Therefore, in this paper a fuzzy logic based cluster head selection algorithm is proposed taking into consideration the fact that the mobility of the client nodes should be seamless from one cluster to another cluster.

In this paper, existing clustering and routing algorithms in wireless networks are discussed in Section 2 whereas Section 3 discusses about the proposed technique. Results and discussions are presented in Section 4 and the conclusion is made in Section 5.

\section{Related Study}

Over the years, various node clustering and routing algorithms are proposed by the researchers mostly for MANETs and WSNs whereas only two clustering schemes are available for WMN.

A good number of schemes are available in the literature which are primarily mobility based, viz, Mobility Prediction-based Clustering (MPBC) [6], Neighborstability based mobility prediction [7], Lowest Relative Mobility Clustering Algorithm (MOBIC) [8], Mobility-based d-hop Clustering Algorithm (MobDHop) [9]. These schemes are proposed for MANETs and WSNs.

The available clustering algorithms for WMNs are Hierarchical Cluster Based Rou ting for WMN [12] and Fuzzy Logic Control Clustering Algorithm (FLCCA) [13].

\subsection{Hierarchical Cluster Based Routing for WMN [12]}

This technique is an enhancement to the Cluster-based Routing Protocol (CRP) $[10,11]$. In CRP, Mesh point portal (MPP) is there on the top of the hierarchy and elects cluster head $(\mathrm{CH})$ and members of each cluster.MPP keeps the information like $\mathrm{CH}$ id, $\mathrm{CH}$ neighbours etc in its own table. All the $\mathrm{CHs}$ have the information about neighboring $\mathrm{CHs}$ and therefore, in the time of routing neighboring $\mathrm{CHs}$ are sent path requests. In this scheme, the MPP is overloaded.

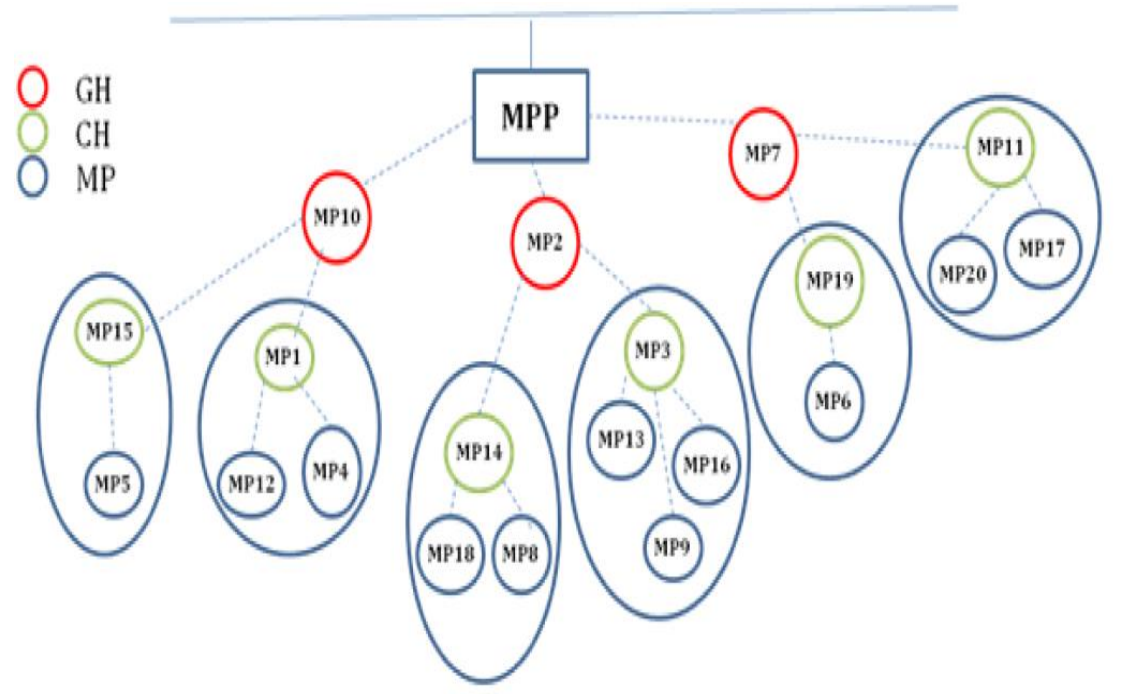

Figure 1. Domains in Wireless Mesh Network

As shown in Figure 1, in the hierarchical cluster based method, a Group Head $(\mathrm{GH})$ is used to control the load on MPP and each GH controls some domains. Under each domain, there are a number of clusters. Each cluster will have one MP as the Cluster head $(\mathrm{CH})$. Here, $\mathrm{CH}$ and MPs maintain the information of all the MPs within the same cluster. Information on its $\mathrm{GH}$ and other $\mathrm{CHs}$ is stored in each 
$\mathrm{CH}$. GH has the information about the MPP and also about the $\mathrm{CHs}$ under its supervision.

Information of all the GHs is maintained by MPP. A single node cannot be considered as both $\mathrm{CH}$ and $\mathrm{GH}$ simultaneously. Big oval shapes in Figure 1 are representing the domains in WMN, three nodes namely, MP10, MP2 and MP7 are considered as GHs. In the Figure, two clusters are maintained by every GH and MP15, MP1, MP14,MP3,MP19 and MP11 are shown as cluster heads.

\subsection{Fuzzy Logic Control Clustering Algorithm (FLCCA)}

In this paper the authors proposed a fuzzy based algorithm to select $\mathrm{CH}$ and gateway for mesh client networks. The method is based on the fuzzification of three parameters, namely, mobility, traffic delivery capacity and cost of service. Due to the traffic pattern of WMNs, Mesh Client (MC) with high traffic delivery capacity has better possibility for getting selected as a $\mathrm{CH}$. Also, the cost of service metric can approximate the message overhead that each MC will produce. So, if the cost of service of an $\mathrm{MC}$ is higher, the less is the chance for selecting it as a $\mathrm{CH}$. Similarly, a highly mobile node creates more re-affiliations and makes the cluster structure further unstable. Therefore, a $\mathrm{CH}$ should be having less mobility. Triangular membership function is used to represent these three parameters and the final fuzzy score for each node is calculated from these three input variables as represented by a trapezoidal membership function. FLCCA requires that every MC should get the fuzzy score value of its one hop neighbour. Once the fuzzy score values are acquired, an $\mathrm{MC}$ with highest score elects itself as $\mathrm{CH}$ among the set of MCs that falls within one-hop of its transmission range. Nodes which reside in the two adjacent clusters having minimal average distance between the two clusters are elected as cluster gateway nodes. The gateway nodes facilitate communication between adjacent clusters.

\section{Proposed Method}

In this paper, we have proposed a fuzzy based hierarchical clustering scheme which considers the hierarchy proposed in [12] where MPP is on the top of the hierarchy having group head $(\mathrm{GH})$ in its lower level and then Cluster head(CH) and on the last level the cluster member MPs. It is assumed that the MPP elects the GHs in and around some particular co-ordinates. GHs are assumed to be static nodes.

\subsection{Fuzzy Input and Output Parameters}

The proposed Fuzzy Logic Controller (FLC) uses four input parameters Node degree, Node Mobility, Distance between GH and MP, Node Residual Power to evaluate the output parameter i.e. the fuzzy score of individual node.

3.1.1. Node Degree: Node degree of a node is the number of 1-hop neighb or of a mesh node. The node which is having maximum number of neighbors in its vicinity is capable of communicating with highest number of nodes in its neighborhood. An MP with higher capacity to transfer data packets should have higher preference to be chosen as $\mathrm{CH}$ because it can decrease control overhead and increase cluster stability in the network. The number of one hop neighbours of $\mathrm{v}_{\mathrm{i}}$ is called the node degree of $v_{i}$ represented as:

$$
n \operatorname{deg}\left(v_{i}\right)=\sum_{d=1}^{n}\left(v_{d}\right)
$$

Node degree membership function is shown in Figure 2(a) as input variable and is made up of three membership functions (Low, Medium and High) which are repre- 
sented as Low [x: $0.0,0.0,0.15,0.5$ ], Medium $[\mathrm{x}: 0.2,0.5,0.8]$, High[x : 0.5, $0.9,1.0,1.0]$.

3.1.2. Node Mobility: Node mobility is the most important parameter in the proposed method. It is assumed that the MP nodes in the WMN may be having low, medium or high mobility. More preference is given to the nodes which are having low mobility for getting selected as $\mathrm{CH}$. Therefore, when fuzzy input variable mobility is defined, it is assumed that the nodes which are actually having moderate mobility may be regarded as having high mobility (Figure $2 \mathrm{~b}$ ). The distance between node $x$ and node $y$ can be found by the formula presented in equation 2 :

$$
\operatorname{Dist}(x, y)=\sqrt{ }\left\{\left(x_{i}-x_{j}\right)^{2}-\left(y_{i}-y_{j}\right)^{2}\right\}
$$

where $\left(x_{i}, y_{i}\right)$ and $\left(x_{j}, y_{j}\right)$ are the coordinates of node $i$ and $j$ respectively. The mobility for each MC from one place to another place is represented as the average speed at current time $t$ as shown in Equation 3 :

$$
M_{v}=1 / T\left\{\sum_{i=1}^{T} \sqrt{ }\left\{\left(x_{i}-x_{i-1}\right)^{2}-\left(y_{i}-y_{i-1}\right)^{2}\right\}\right.
$$

Node mobility input variable is made up of three membership functions (Low, Medium and High). The variables are organized as Low $[x: 0.0,0.0,0.2,0.4]$, Medium $[x: 0.2,0.4,0.6]$, High $[x: 0.5,0.8,1.0,1.0]$.

3.1.3. Distance between Group Head and MP (DGM): The distance between MP and GH is also a very important criterion for hierarchical clustering. The MP which is nearer to the GH has better chance to deliver the packets to the MPP with minimum error. The distance can be found by using Equation $(2)$, where $\left(x_{i}, y_{i}\right)$ is the coordinate of mesh point and $\left(x_{j}, y_{j}\right)$ may be the co-ordinate of GH. The membership function of DGM as shown in Figure $2 \mathrm{c}$ is also defined similar to the node degree variable i.e., with three values having similar ranges.

3.1.4. Residual Energy: Every node is capable of measuring its own residual energy. Residual energy is presented by 3 triangular membership functions as shown in Figure 2(d). It is also same as Node degree and DGM. All these three variables are represented by triangular membership function specified by three parameters $\left\{a_{1}, a_{2}\right.$, $\left.a_{3}\right\}$ as follows:

$$
\operatorname{triangular}\left\{\mathrm{x}: \mathrm{a}_{1}, \mathrm{a}_{2}, \mathrm{a}_{3}\right\}=\left[\begin{array}{cc}
0 & \mathrm{x}<\mathrm{a}_{1} \\
\left(\mathrm{x}-\mathrm{a}_{1}\right) /\left(\mathrm{a}_{2}-\mathrm{a}_{1}\right) & \mathrm{a}_{1} \leq \mathrm{x} \leq \mathrm{a}_{2} \\
\left(\mathrm{a}_{3}-\mathrm{x}\right) /\left(\mathrm{a}_{3}-\mathrm{a}_{2}\right) & \mathrm{a}_{2} \leq \mathrm{x} \leq \mathrm{a}_{3} \\
0 & \mathrm{x}>\mathrm{a}_{3}
\end{array}\right]
$$



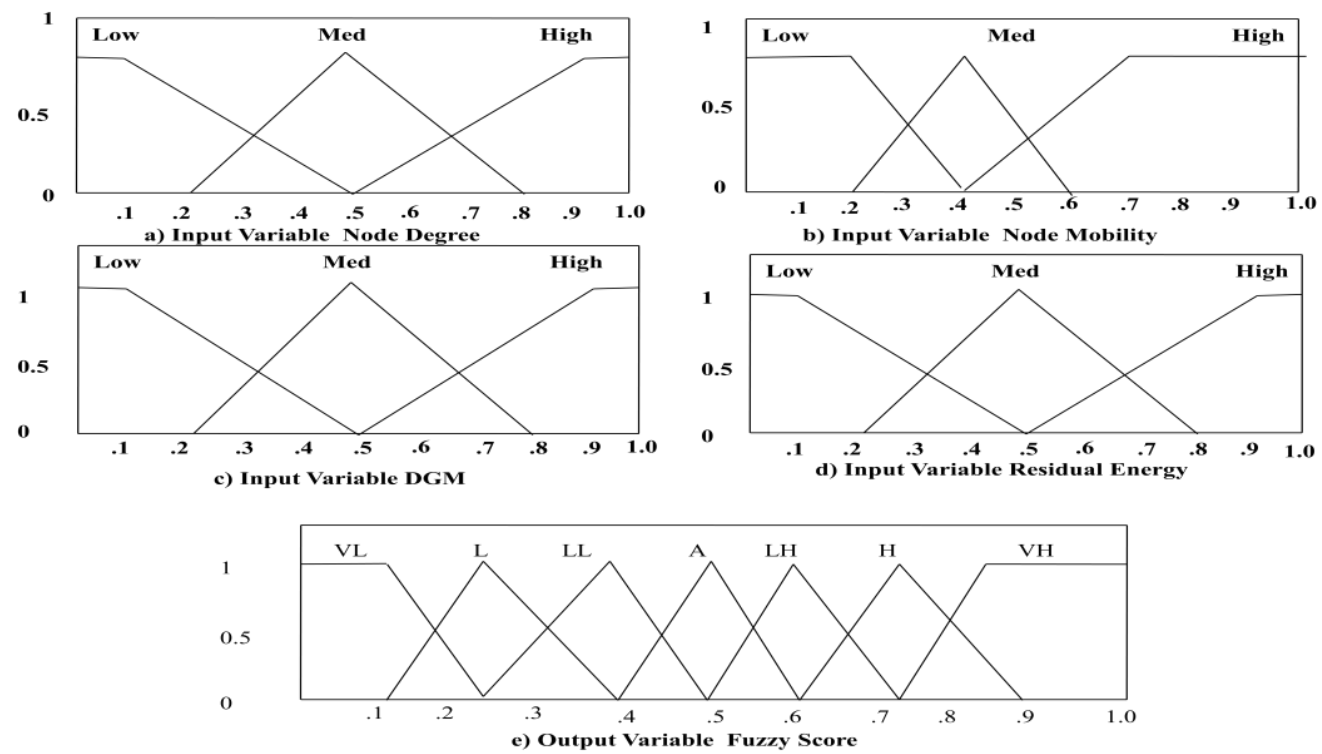

Figure 2. Fuzzy Input and Output Variables

3.1.5. Output Fuzzy Score: Output fuzzy score of each node that gives the final value for MPs to determine its suitability for election as $\mathrm{CH}$ is an output variable that consists of seven membership functions [VeryLow (VL), Low (L), LittleLow (LL), Average (A), LittleHigh ( $\mathrm{LH})$, High(H), and VeryHigh $(\mathrm{VH})]$ respectively (Figure 2e). The variables are represented as follows: VL $[x: 0.0,0.0,0.1,0.2], \mathrm{L}[x$ $: 0.1,0.25,0.4], \mathrm{LL}[x: 0.25,0.4,0.5], \mathrm{A}[x: 0.4,0.5,0.6], \mathrm{LH}[x: 0.5,0.6,0.7], \mathrm{H}$ $[x: 0.6,0.7,0.9], \mathrm{VH}[x: 0.7,0.85,1.0,1.0]$. The output variable fuzzy score is represented by trapezoidal membership function specified by four parameters $\left\{a_{1}, a_{2}\right.$, $\left.a_{3}, a_{4}\right\}$ as follows

trapezoidal $\left\{\mathrm{x}: \mathrm{a}_{1}, \mathrm{a}_{2}, \mathrm{a}_{3}, \mathrm{a}_{4}\right\}=\left(\begin{array}{cc} & \mathrm{x}<\mathrm{a}_{1} \\ 0 & \mathrm{a}_{1} \leq \mathrm{x} \leq \mathrm{a}_{2} \\ \left(\mathrm{x}-\mathrm{a}_{1}\right) /\left(\mathrm{a}_{2}-\mathrm{a}_{1}\right) & \mathrm{a}_{2} \leq \mathrm{x} \leq \mathrm{a}_{3} \\ 1 & \mathrm{x}>\mathrm{a}_{4}\end{array}\right)$

\subsection{Fuzzy Logic Inference Engine and Fuzzy Rule Base}

Mamdani fuzzification model is used in our proposed method as was applied in [14] by Gupta et al to find the $\mathrm{CH}$ in sensor networks. Figure 3 shows the general Fuzzy logic controller structure with fuzzifier, Fuzzy Inference Engine, Fuzzy Rule Base and defuzzifier. Firstly, the crisp input is fuzzified using predefined membership functions. Then the Fuzzy Rule Base is applied to the fuzzified input. The crisp out- put is finally generated by applying defuzzification to the output of the inference engine. 


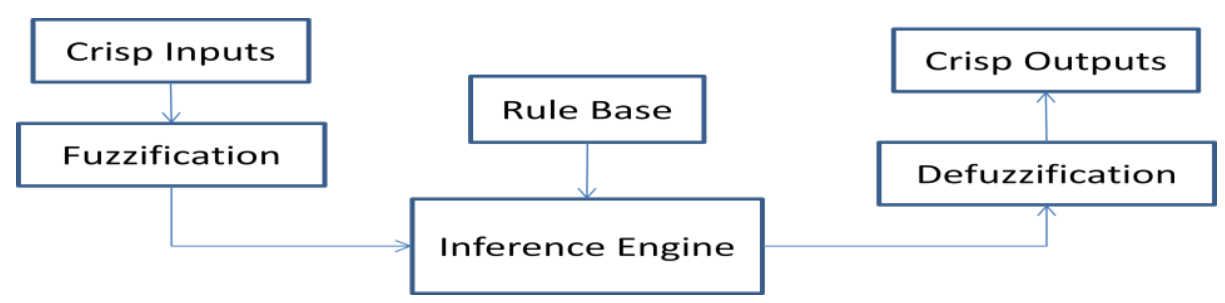

Figure 3. Fuzzy Logic Controller (FLC) Structure

Table 1. Fuzzy Rule Base

\begin{tabular}{|c|c|c|c|c|c|}
\hline $\begin{array}{c}\text { Sl. } \\
\text { no. }\end{array}$ & $\begin{array}{l}\text { Node de- } \\
\text { gree }\end{array}$ & $\begin{array}{c}\text { Residual } \\
\text { Energy }\end{array}$ & DGM & $\begin{array}{c}\text { Node Mo- } \\
\text { bility }\end{array}$ & $\begin{array}{l}\text { Fuzzy } \\
\text { Score }\end{array}$ \\
\hline 1 & H & H & L & L & VH \\
\hline 2 & H & H & L & M & A \\
\hline 3 & H & H & L & H & VL \\
\hline 4 & H & H & M & L & H \\
\hline 5 & H & H & M & M & LL \\
\hline 6 & H & H & M & H & VL \\
\hline 7 & H & H & H & L & LH \\
\hline 8 & H & H & H & M & L \\
\hline 9 & H & H & H & H & VL \\
\hline 10 & H & M & L & L & VH \\
\hline 11 & H & M & L & M & A \\
\hline 12 & H & M & L & H & VL \\
\hline
\end{tabular}

The inference engine takes the decisions like the human reasoning process by applying IF-THEN rules from the fuzzy rule base. There are $81(3 \times 3 \times 3 \times 3)$ rules in our fuzzy rule base as our every fuzzy input variable has 3 values (low, medium \& high).Table 1 presents some rules that were designed for the fuzzy inference engine which takes the form IF $u$ AND $v$ AND $w$ AND $x$ THEN $z$. The $u, v, w, x, z$ represent node degree, residual energy, distance between MP \& GH (DGM), node mobility and output fuzzy score respectively. Here, utmost importance is given to node mobility as mentioned before. Therefore, whenever there is a high mobility node, there will be very little chance for that node to be selected as $\mathrm{CH}$.

\subsection{Defuzzification}

After fuzzification, the input data are converted into linguistic values .Now, the defuzzification step will be used to convert the range of output values into Crisp output values. In the defuzzification process, we would like to represent the possibility distribution of an inferred fuzzy control action to a non-fuzzy control action [15]. In the proposed scheme, centroid defuzzification method is used. This method finds out the center of area below the curve considered. 


\subsection{Clusterhead Selection and Cluster Maintenance Algorithm}

\section{Pseudocode for Proposed Method.}

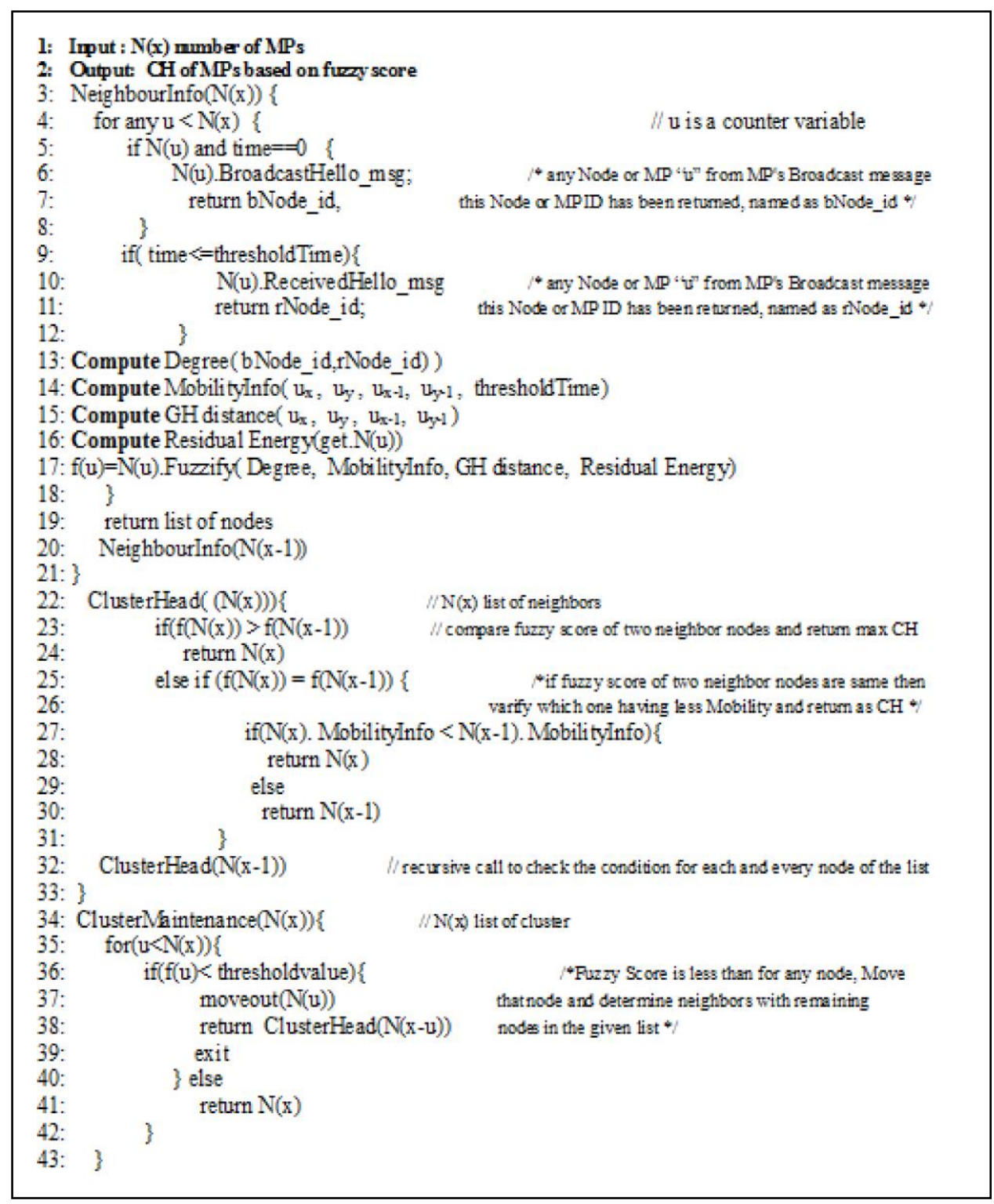

The pseudo code of proposed algorithm for $\mathrm{CH}$ selection and maintenance is given above. Based on the received hello message and response, we can generate list of neighbors for each node. Then we can find the fuzzy score of each node by applying the above algorithm. For determining $\mathrm{CH}$, we find the maximum fuzzy score of a mesh node from the list of neighbours and elect this mesh node as a $\mathrm{CH}$ of this given neighbour list. In any circumstance, if two clients are having same fuzzy score within a list then identify the MP having less mobility and select that node as a $\mathrm{CH}$. To maintain the cluster, we determine fuzzy score of all the nodes in a cluster and determine which client is having fuzzy score less than threshold value and move out that client from that cluster and find the cluster head from the remaining nodes. 


\section{Results and Discussion}

The proposed clustering algorithm is simulated using NS3 network simulator. A number of MPs are simulated within a network area of 1000x1000 square meters. FLCCA [13] is also simulated in the same environment and the results are compared with the proposed one. Three performance evaluation parameters were checked, namely, number of cluster formed, stability of clusters and overhead. The different parameters of simulation environment of our experiment are given in Table 2.

Table 2. Simulation Environment

\begin{tabular}{|c|c|}
\hline Parameter & Value \\
\hline Simulator & NS 3.24 \\
\hline Network Area & $1000 \mathrm{~m}$ x 1000m \\
\hline Number of Nodes & $20-150$ \\
\hline Simulation Time & $382 \mathrm{sec}$ \\
\hline Mobility Model & $\begin{array}{c}\text { Random Direction } \\
\text { 2D model }\end{array}$ \\
\hline Node placement & Random \\
\hline Transmission Range & $50-100 \mathrm{~m}$ \\
\hline Mobility Speed & $0-20 \mathrm{~m} / \mathrm{s}$ \\
\hline Packet Size & 512 bytes \\
\hline Buffer Size & $50 \mathrm{~B}$ \\
\hline
\end{tabular}

\section{1. no. of Clusterheads (CHs)}

Number of CHs with increased node density (Figure 4) shows better result for our algorithm compared to the FLCCA. The number of $\mathrm{CH}$ decreases with the increase in transmission range (Figure 5) and the proposed method performs better.

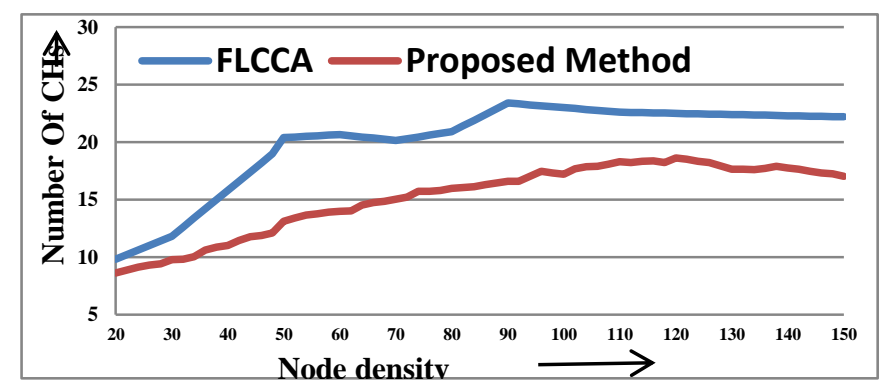

Figure 4. Node density Vs Number of Clusterheads (CH)

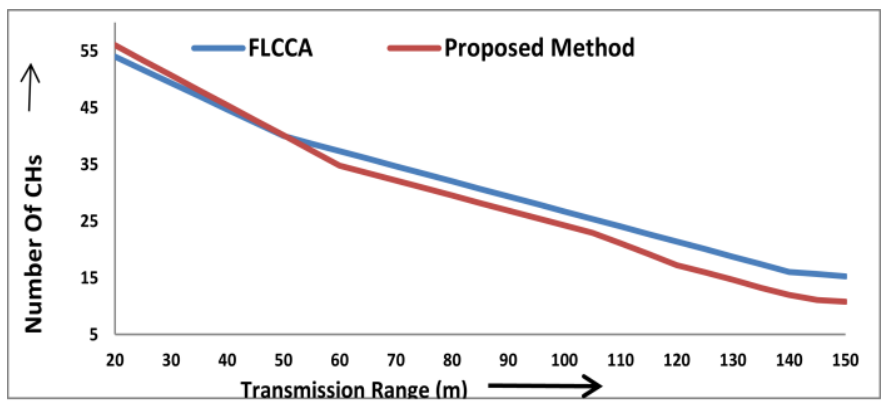

Figure 5. Transmission Range Vs Number of Clusterheads (CH) 


\subsection{Cluster Stability}

Cluster stability is also evaluated for both the algorithms. The mobility speed of the mesh nodes is changed to find the simulation results in Figure 6. With increasing mobility of the nodes the proposed algorithm shows slightly better result.

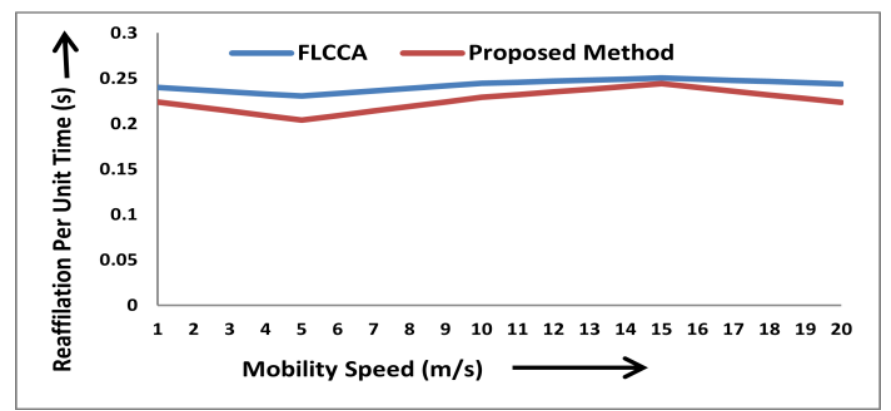

Figure. 6. Mobility Speed Vs Number of Re-affiliations

\subsection{Clustering Overheads}

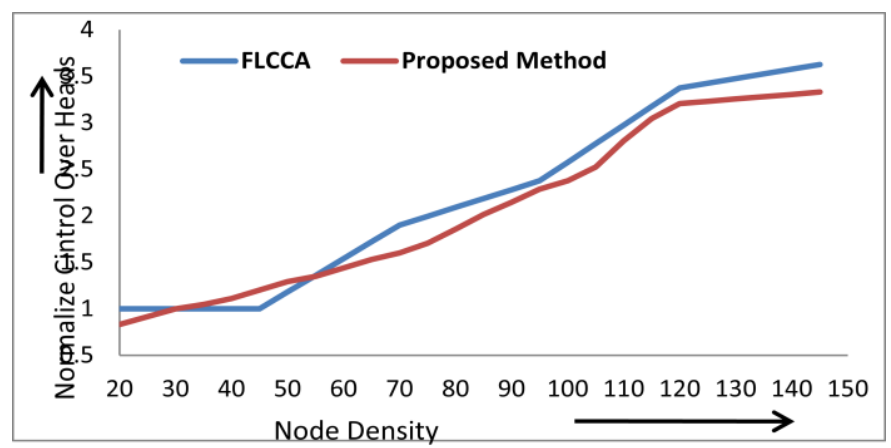

Figure 7. Node Density Vs Control Overhead

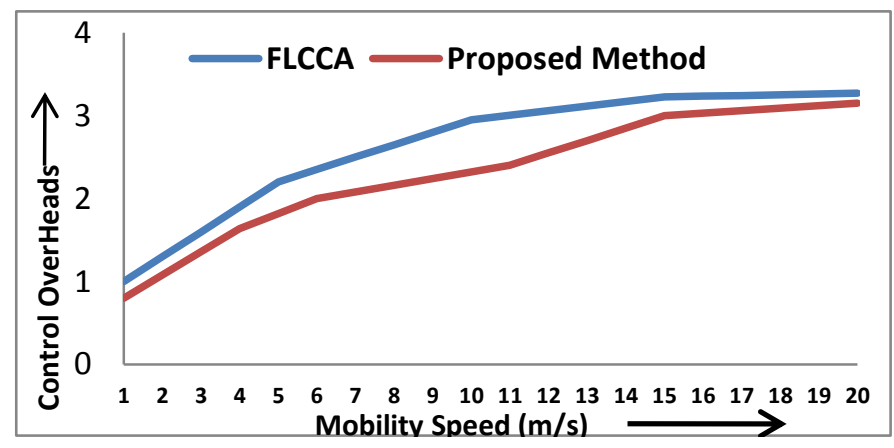

Figure 8. Mobility Speed Vs Control Overhead

An increase in node density results in an increase in clustering overheads (Figure 7). This is happening because with increasing number of nodes, traffic generated by the MPs also increase due to the exchange of different messages like Hello messages for initialization, cluster update and maintenance. Increase in mobility speed (Figure 8) increases the overhead a bit as re-affiliation chances may increase slightly. In both the cases the proposed method shows slightly better results. 


\section{Conclusion}

In this paper, a new fuzzy based node clustering technique for wireless mesh network is proposed. The method works with four variables: node degree, node mobility, distance between GH and MP and residual energy of MP. The proposed algorithm is implemented in NS3.The results are compared with FLCCA algorithm and the method gives slightly better result in all the performance evaluation parameters. In future, the membership function values may be changed as well as some new parameters may also be checked to find out the change in the output. Other soft computing techniques may also be explored to find out best results.

\section{References}

[1] I. F. Akyildiz, X. Wang and W. Wang, "Wireless mesh networks: a survey", Computer Networks, vol. 47, no. 4, (2005), pp. 445-487.

[2] Z. Yan, L Jijun and H. Hongli, "Wireless Mesh Networking, Architectures Protocols and Standard", Auerbach Publications, USA, (2007).

[3] Y. Y. Su, S. F. Hwang and C. R. Dow, "An Efficient Cluster-Based Routing Algorithm in Ad Hoc Networks with Unidirectional Links", Journal of Information Science and Engineering, vol. 24, no. 5, (2008), pp.1409-1428.

[4] R. Agarwal and M. Motwani, "Survey of Clustering Algorithms for MANET", International Journal on Computer Science and Engineering, vol. 1, no. 2, (2009), pp. 98-104.

[5] S. Chinara and S. K. Rath, "A Survey on One-Hop Clustering Algorithms in Mobile Ad Hoc Networks”, Journal of Network System Management, vol. 17, no. 1, (2009), pp. $183-207$.

[6] M. Ni, Z. Zhong and D. Zhao, "MPBC: A Mobility Pre-diction-Based Clustering Scheme for Ad Hoc Networks", IEEE TVT, vol. 60, no. 9, (2011), pp. 4549 - 4559.

[7] C. Konstantopoulos, D. Gavalas and G.Pantziou, "Clustering in Mobile Ad Hoc Networks through Neighbor-hood Stability-based Mobility Prediction”, Computer Networks Journal, vol. 52, (2008), pp. 1797-1824.

[8] P. Basu, N. Khan, and T. D. C. Little, "A Mobility Based Metric for Clustering in Mobile Ad Hoc Networks", ICDCSW, (2001), pp. 413-418.

[9] A. H. Hussein, A. O. A. Salem and S. Yousef, "A flexible Weighted Clustering Algorithm Based on Battery Power for Mobile Ad hoc Networks", in Proceedings of IEEE International Symposium Industrial Electronics (ISIE), Cambridge, UK, (2008).

[10] M. Singh, S. G. Lee, T. W. Kit, and L. J. Huy, "Clusterbased routing scheme for Wireless Mesh Networks", 13th International Conference on Advanced Communication Technology (ICACT), Phoenix Park, Republic of Korea, (2011).

[11] M. Singh, S. G. Lee and D. Singh, "A Simulation-Based Performance Analysis of a Cluster-based Routing Scheme for Wireless Mesh Networks", International Journal of Multimedia and Ubiquitous Engineering, vol. 8, no. 5, (2013), pp.283-296.

[12] D. Kaushal, G. Niteshkumar, B. Prasann and V. Agarwal, "Hierarchical cluster based routing for wireless mesh networks using group head", International Conference on Computing Sciences (ICCS), Phagwara, Punjab, India, (2012).

[13] A. Adekiigbe, K. Abu-Bakar, B. M. G. Amosa and N. H. Umelo, "Fuzzy Logic Approach to Clustering Algorith for Mesh Client Networks", Application of Information and Communication Technologies to Teaching, Research and Administration, Obafemi Awolowo University, Ile Ife, Nigeria, (2013).

[14] I. Gupta, D. Riordan and S. Sampalli, "Clusterhead election using fuzzy logic for wireless sensor networks", Proceedings of Communication Networks and Services Research Conference, Halifax, Canada, (2005).

[15] K. H. Lee, "First Course on Fuzzy Theory and Applications", Springer, Germany, (2005).

\section{Authors}

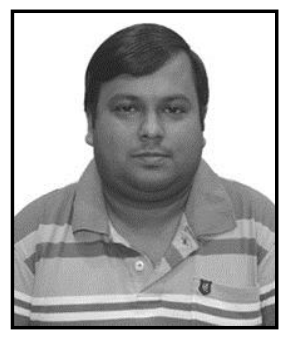

Tapodhir Acharjee, he is working as an Assistant Professor in the Department of Computer Science \& Engineering, Assam University, Silchar, India. He received his M.Tech. degree from Tezpur University, Assam, India in 2008. Currently he is pursuing his $\mathrm{PhD}$ from Assam University. His areas of interests include Wireless Ad-hoc Networks and Cryptography \& Network Security. 
Sudipta Roy, he is a Professor in the Department of Computer Science and Engineering, Triguna Sen School of Technology, Assam University, Silchar, Assam,India. His research interests are image processing, soft computing techniques and its applications, wireless networking and network security. He has published numerous papers in reputed international and national journals and conference proceedings. He is member of LMCSI, SMIEEE(USA), SMIACSIT, MIE, MINNS(USA), LMISTE. 
International Journal of Future Generation Communication and Networking Vol. 9, No. 12 (2016) 